\title{
What does shared decision making look like in natural settings? A mixed methods study of patient-provider conversations
}

Author: Joy Lee

Affiliations: Indiana University School of Medicine, Indianapolis, USA; Regenstrief Institute, Inc., Indianapolis, USA; Johns Hopkins Bloomberg School of Public Health, Baltimore USA Full address: Regenstrief Institute, 1101 W. $10^{\text {th }}$ St. Indianapolis, IN 46202, USA

Email: joyllee@iu.edu

Author: Wynne Callon

Affiliation: Johns Hopkins School of Medicine, Baltimore, USA

Full address: Johns Hopkins University School of Medicine, Baltimore, MD 21202, USA.

Email: wcallon1@jhmi.edu

Author: Carlton Haywood, Jr.

Affiliation: Johns Hopkins School of Medicine, Baltimore, USA

Full address: 2024 E. Monument Street, Suite 2-600, Baltimore, MD 21205, USA

Email: chaywoodjr@jhu.edu

Author: Sophie M. Lanzkron

Affiliation: Johns Hopkins School of Medicine, Baltimore, USA

Full address: 1830 E Monument Street, Suite 7300, Baltimore, MD 21205, USA

Email: slanzkr@jhmi.edu

This is the author's manuscript of the article published in final edited form as:

Lee, J., Callon, W., Carlton Haywood, J., Lanzkron, S. M., Gulbrandsen, P., \& Beach, M. C. (2018). What does shared decision making look like in natural settings? A mixed methods study of patient-provider conversations. Communication \& Medicine, 14(3), 217-228. https://doi.org/10.1558/cam.32815 
Author: Pål Gulbrandsen

Affiliation: University of Oslo, Oslo, Norway; Akershus University Hospital, Lørenskog,

Norway

Full address: HØKH, Research Centre, Akershus University Hospital, Mail drawer 95,

Lørenskog 1478, Norway

Email: pal.gulbrandsen@medisin.uio.no

Author: Mary Catherine Beach

Affiliation: Johns Hopkins Berman Institute of Bioethics and Johns Hopkins School of Medicine,

Baltimore, USA

Full address: 2024 East Monument Street, Room 2-500, Baltimore, MD 21205, USA

Email: mcbeach@jhmi.edu 


\section{Abstract}

Objective: To understand the variability and nature of shared decision making (SDM) regarding a uniform type of serious medical decision, and to make normative judgments about how these conversations might be improved.

Methods: This was a mixed-methods sub-analysis of the Improving Patient Outcomes with Respect and Trust (IMPORT) Study. We used the Braddock framework to identify and describe seven elements of SDM in audio-recorded encounters regarding initiation of hydroxyurea, and used data from medical records and patient questionnaires to understand whether and how these tasks were achieved.

Results: Physicians covered a spectrum of SDM behaviors: all dialogues contained discussion regarding the clinical issue and the pros and cons of treatment; the patient's understanding and role were not explicitly assessed or stated in any encounter. Yet no patient agreed to start hydroxyurea who did not already prefer it. There was no uniform approach to how physicians presented risk; many concerns expressed by patients in a pre-visit questionnaire were not discussed.

Conclusion: In this analysis, patients seemed to understand their role in the decision-making process, suggesting that a patient's role may not always need to be explicitly stated. However, shared decision making might be improved with more routine assessment of patient understanding and concerns. Standardized decision aids might help fully inform patients of risks and benefits. 
Keywords: shared decision making, patient-provider communication, sickle-cell disease, conversation analysis, SDM framework

\section{Introduction}

Much has been written about the principles surrounding shared decision making (SDM), defined as an approach to making decisions through which patients and physicians "share the best available evidence [and] where patients are supported to consider options to achieve informed preferences" (Elwyn et al. 2012: 1361). Shared decision making is governed by ethical principles of respect for patient autonomy and acknowledgement of patients' rights to selfdetermination, and is aligned with patient-centeredness as a core element of healthcare quality (Elwyn et al. 2012). While there is widespread support for SDM in principle, studies that have examined it in practice have tended to document extreme deficiencies in the extent to which it occurs (Braddock et al. 1999; Elwyn et al. 2001). Gulbrandsen et al. (2014), for example, highlight confusion regarding how to carry out SDM from physicians who were not trained in SDM, despite above average patient-centered skills.

Few studies have specifically tried to bridge the gap between the literature that demonstrated how providers fall short and the literature that closely examines the dialogue itself. In this paper, we seek to understand the nature of SDM during a series of discussions regarding a uniform type of serious medical decision. In doing so, we hope to examine the context of each conversation to understand why providers do not achieve the standards, and then advance the field of shared decision making with normative judgments about what it ought to consist of and how it might be realistically improved. The results of this paper will be of interest to a number of stakeholders, including patients, physicians, and health communication researchers. 
In this paper, we describe the presence and absence of shared decision making using the Braddock framework to characterize conversations between patients with sickle-cell anemia and their providers. From this application, the analysis then broadens to consider the decisions reached as a result of these conversations. To further contextualize these conversations, our analysis also considers the relationship between patient characteristics and treatment decisions, with attention to patient concerns raised and not raised in these interactions

\section{Literature review}

Despite the mounting scholarship on shared decision making, particularly on specific aspects of SDM, and acknowledgement of the need to consider SDM in context of the patientprovider relationship, what remains uncertain from this body of literature is why so many wellintended clinicians fail to meet such a basic ethical requirement. Some researchers have looked closely at shared decision making dialogues and have identified interactional phenomena and strategies used by providers. In one analysis, Landmark et al. pinpointed how physicians summarize patient preferences as a strategy for reconciling treatment preferences and bringing patient and physician stances closer together (Landmark et al. 2016). Other researchers have focused on the directionality of decision making (Collins et al. 2005; Pilnick and Zayts 2016). While some have conceptualized traditional decision making as physician driven and unilateral (i.e. physician deciding for patient) and SDM as a bilateral process (i.e. physicians and patients deciding together), Collins and colleagues, and Pilnick and Zayts, found decision making to occur on a continuum and that the presence of unilateral and bilateral decision making to be dependent on context (Collins et al. 2005; Pilnick and Zayts 2016). "Characteristics and qualities 
of a 'bilateral' approach," Collins and colleagues noted, "may tend to vary across clinical situations" (Collins et al. 2005: 2626).

Indeed, the inclination to consider SDM without a fuller clinical context may explain why so many providers fall short of the SDM ideal (Matthias et al. 2013). Robertson et al. suggest that SDM should not be approached simply as a desired outcome, but should be considered in the context of the richness and complexity of the patient-provider relationship, from the conversational forms to make up the discussions (Robertson et al. 2011) to the power asymmetry inherent in how medicine is structured (Pilnick and Dingwall 2011) to the concept of decision ownership (Mendick et al. 2010). Decision, then, is context dependent, and includes both subjective elements (how patients see the decision-making process) and procedural elements (whether a patient is told of treatment options) (Mendick et al. 2010). Our analysis attempts to understand the elements of SDM and uses pre-encounter questionnaires and patient health information to inform the context of a single conversation.

\section{Methods}

We used a mixed methods study design and focused our analysis on conversations between physicians and patients with sickle cell disease (SCD) regarding the decision to start hydroxyurea (HU). Sickle cell disease is a serious genetic condition associated with severe, disabling and unpredictable episodes of acute pain, organ failure, chronic pain, and early mortality. Hydroxyurea is the only Food and Drug Administration (FDA)-approved medication to treat SCD. There are no other pharmacological interventions that treat the underlying disease but the alternative treatments can ameliorate the symptoms of SCD (National Heart, Lung, and 
Blood Institute 2014). Alternatives to hydroxyurea exist in the form of more aggressive treatments such as chronic blood transfusion and bone marrow transplant.

However, many patients who would benefit from $\mathrm{HU}$ choose not to take it due to concerns about side effects (e.g. birth defects if pregnant, lowered blood counts that improve after stopping the drug, and a very small or theoretical risk of malignancy).

\subsection{Study Design, Subjects and Setting, and Data Collection Methods}

This analysis was a sub-study of the Improving Patient Outcomes with Respect and Trust (IMPORT) Study, a 3-year cohort study of 291 SCD patients, which took place at two academic health centers in the mid-Atlantic region. In the IMPORT Study, SCD clinicians who saw adult or adolescent patients agreed to participate and gave written informed consent at both institutions. Patients of participating clinicians were then enrolled when they presented for a clinical appointment at one of the participating health centers. Patients then completed baseline questionnaires with measures of the patient's desired role in decision-making, current medications (including use of and concerns about hydroxyurea) and number of hospitalizations per year. No baseline information was collected from clinicians. Following the questionnaire, a routine visit with their usual SCD clinician was audio-recorded and transcribed. All study procedures were reviewed by the Institutional Review Board at both institutions.

\subsection{Patient Inclusion Criteria}

From the database of audio-recorded baseline patient-physician encounters, we identified the encounters in which communication coders indicated that there was some discussion of hydroxyurea in the dialogue among patients who reported not being on hydroxyurea. Of these 43 
encounters, we excluded 29 because there were only brief mentions and no substantive discussions about starting treatment that day. We focused on 14 encounters where the decision to start hydroxyurea was substantively discussed with a patient not currently taking the medication.

\subsection{Analysis of Dialogue and Elements of Shared Decision Making}

We used the framework set forth by Braddock et al. (hereafter the Braddock framework) to identify and describe how SDM occurred in these audio-recorded encounters (Braddock et al. 1999). The seven elements of the framework are: (1) specifying the patient's role in decision making; (2) a description of the clinical issue; (3) an explanation of the alternatives; (4) the pros and cons of treatment and alternatives; (5) the uncertainties surrounding the decision; (6) an assessment of patient understanding; and (7) an exploration of patient preference.

The Braddock framework was developed based on ethical consensus about the nature of SDM and is therefore normative, meaning that a pre-specified number of discussion elements must be present for a discussion to count as complete. In their initial study that took place in the primary care setting, Braddock et al. considered that there were different levels of decision complexity in terms of effect on the patient, medical consensus, and nature of outcome and proposed that more basic decisions ethically required a smaller set of SDM discussion elements compared to more complex decisions in order to count as "complete".

In our use of the framework, we adopted the same criteria for whether or not a discussion element counted as present or absent, but we did not necessarily make normative judgments about whether the SDM discussion was complete or incomplete based on which elements occurred. Although we did not have a specific disagreement with Braddock et al.'s criteria, we did not wish to specify in advance which discussion elements were ethically required. Rather, we 
used the framework to describe, in this clinical setting with this population of patients, how decision making in the context of a serious, chronic, and life-threatening illness takes place. In this way, we hoped to learn what gets discussed and how it gets discussed, in order to then make a judgment about what might be ethically required under these circumstances.

Two reviewers (JLL and MCB) coded and discussed each encounter for dialogue relating to each of the seven elements, coming to consensus about whether or not that dialogue counted as a complete fulfillment of the element based on the Braddock et al. study. During this iterative process, we added the 'partially fulfilled' category to account for variations and nuances found in the dialogue. Once all examples of a given discussion element were identified, we both read and compared across the encounters how that element was discussed. We highlighted examples of each element to demonstrate the variations and nuances that exist in shared decision making.

Finally, we ascertained from the dialogue whether or not the patient and the physician were in favor of, ambivalent about, or against starting hydroxyurea. By mapping patient and provider preferences with decision outcomes, we explored trends in how decisions to start hydroxyurea are made.

\subsection{Contextual Data}

Our analysis also incorporated data from the baseline patient survey, including number of hospitalizations in the past year and patients' concerns regarding hydroxyurea (if any). Data about hospitalizations provide insight into how much benefit the patient would gain from hydroxyurea, since it is more strongly recommended for those with 3 or more hospitalizations per year (National Heart, Lung, and Blood Institute 2014). The patient-reported concerns helped 
us understand the extent to which physicians elicited or patients brought up their specific concerns.

\section{Results}

\subsection{Patient and Provider Characteristics}

Fourteen patient-provider encounters were included in this analysis; the patients were all African American, and 10 (71\%) were female (see Table 1). The mean patient age was 31.5 years with a range of 15-64. Eight patients $(57 \%)$ reported $<2$ hospitalizations for acute pain over the past year, while $5(36 \%)$ reported 3-5 hospitalizations for acute pain (suggesting a clinical indication for $\mathrm{HU}$ ), and one patient (7\%) had >6 hospitalizations (suggesting stronger clinical indication). Seven primary SCD providers were included who interacted with the patients (5 physicians and 2 nurse practitioners).

Table 1. Study Participant Characteristics

\begin{tabular}{lc}
\hline Characteristic & $\begin{array}{c}\text { Participants } \\
(\mathbf{n}=\mathbf{1 4})\end{array}$ \\
\hline Mean age (range) & $31.5(15-64)$ \\
Female, \% & $71 \%$ \\
African American, \% & $100 \%$ \\
Hospitalizations for acute pain over past year, $\%$ & \\
$<2$ & $57 \%$ \\
$3-5$ & $36 \%$ \\
$\geq 6$ & $7 \%$ \\
\hline
\end{tabular}

\subsection{Elements of Shared Decision Making}

Among the 14 dialogues, physicians initiated all but one of the conversations regarding initiation of HU. In some encounters, HU was introduced for the first time while in others, the conversation was clearly following up on prior discussions. Table 2 describes the presence or 
absence of each discussion element in the encounters while the following section describes in qualitative detail how each element occurred.

Table 2. Frequency of Clinician Shared Decision Making Behaviors in Hydroxyurea Initiation Dialogue

\section{Fully fulfilled $\quad$ Not fully fulfilled}

\begin{tabular}{lcccc} 
& $\begin{array}{c}\text { Explicit } \\
\text { Fulfilment }\end{array}$ & $\begin{array}{c}\text { Patient offers } \\
\text { unprompted }\end{array}$ & $\begin{array}{c}\text { Implicit or Partial } \\
\text { Fulfilment }\end{array}$ & No fulfillment \\
\hline Patient's role & 0 & - & 2 & 12 \\
Clinical issue & 14 & - & - & 0 \\
Alternatives & 7 & - & 2 & 0 \\
Pros and cons & 11 & - & 3 & 8 \\
Uncertainties & 6 & - & - & 11 \\
Patient understanding & 0 & 1 & 2 & 7 \\
Patient preference & 3 & 3 & 1 &
\end{tabular}




\subsection{Nature of Shared Decision Making}

Shared Decision Making can take many forms. In the following we apply and discuss each element of the Braddock framework in relation to the conversations we observed.

\subsubsection{Patient Role}

A discussion of the patient's role in the decision making process acknowledges and empowers the patient as a participant. No encounter in the analysis included an explicit discussion by the physician of the patient's role. However, two physicians did assign roles to their patients, and in so doing, implied their inclusion in the process. For example, one physician concluded the discussion by saying:

Example 1

"Then the homework for both of you is to [read] about hydroxyurea and then I could write down [a couple of websites] for you." (Encounter 05)

By assigning 'homework,' the physician verbalizes and acknowledges the patient's participation in this decision.

\subsubsection{Clinical Issue}

A discussion of the clinical issue prompting the decision provides patients with a context for the subsequent conversation. This was the one element of shared decision making that all 14 encounters in the analysis included explicitly. Here, for example, one physician succinctly summarizes how hydroxyurea may be helpful to a patient:

\section{Example 2}

"So there's a medicine that is being used in people with sickle cell called Hydroxyurea. And this is a capsule that you take every day. And what it 
seems to do for-for most people, is that on average, your chances of getting

a pain crisis is cut in half. (Encounter 10)

\subsubsection{Alternatives}

When physicians present hydroxyurea to patients, the Braddock model suggests that other choices available to patients should also be explicitly discussed. While hydroxyurea is the only FDA-approved medication available to prevent sickle cell crises, we broadly considered a full discussion of alternatives to include mention of some other treatments or explicitly considering not taking hydroxyurea. In one encounter, the physician offers HU and then potential future alternatives depending on circumstances.

Example 3

"You can be on hydroxyurea," one physician counsels, “and when you're seriously thinking about having babies then you can go off hydroxyurea, and we can then put you in an exchange transfusion program” (Encounter 12).

Half of the encounters $(n=7)$ explicitly discussed the alternatives while an additional two discussed the lack of other medications for sickle cell anemia but did not touch upon other treatments or not taking hydroxyurea as alternatives (these were considered as only partially fulfilled). The remaining five did not discuss alternatives at all.

\subsubsection{Pros and Cons}

According to Braddock et al. (1999), a full discussion of treatment should encompass not just the potential benefits but the risks as well. All 14 encounters included a discussion of the potential benefits of hydroxyurea. In three encounters, no side effects were discussed. We considered those encounters to have an incomplete discussion of risks and benefits, whereas the 
remaining 11 discussions were considered to have met the criteria fully, despite the variability in discussions described below.

\subsubsection{Benefits}

The 14 encounters included patients with varying degrees of disease severity and physicians with different experiences with HU. Because of this variation, both physician perspectives on whether hydroxyurea is appropriate for the patient and patient preference for the medication differed between encounters. This ranged from vaguely suggesting that hydroxyurea "helps patients who have $S$-S and help children who have $S$-C" to detailing its many potential positive effects on the patient, as this physician did:

Example 4

"I think that it would probably help with the growth, energy and appetite. You don't have so much pain from your sickle cell disease that I think you need to be on it for the pain, but it probably would [still] decrease how much pain you have. “ (Encounter 5)

Hydroxyurea's ability to reduce the frequency of pain crises was the most frequently mentioned benefit -8 of the 14 encounters discussed this effect. The potential reduction in pain more generally was another benefit that came up in several conversations $(n=5)$.

\subsubsection{Risks}

Potential side effects were the foremost concern for patients in the discussions. Almost all of the risk discussions mentioned the possibility of low blood count $(n=8)$ as a part of the mechanism of how hydroxyurea works and the need for close monitoring. Five conversations mentioned the risk of reproductive-related issues, such as low sperm count and birth defects; physicians explained that the risk was low and patients should be on birth control if they take hydroxyurea: 
Example 5

"There is a risk that it might affect the babies... But I can assure you that, number one, while you are on hydroxyurea you're supposed to use protection if you are sexually active. ...[We've] had a few of our patients who have had babies while they are on hydroxyurea and so far we haven't had any problems with their babies. So it's a slight risk [but] we don't want it on the plate at all." (Encounter 12)

Although use of hydroxyurea has not been associated with cancer in SCD patients, the subject of cancer came up in 6 out of the 10 risk conversations. The subject was mostly initiated by the physician (5/6 encounters). For example, one physician explained it this way:

Example 6

"People talk about cancers when they take hydroxyurea. There's a lot of information out there. We looked at this, we studied it really hard in patients that have sickle cell disease, and we didn't see any extra cancers at all in the patients with sickle cell disease." (Encounter 8)

These conversations about cancer suggest that even though there is no documented increased cancer risk associated with hydroxyurea use for these patients, physicians (or patients) are cognizant of a perception that HU is associated and are concerned enough about this perception to discuss the connection. In addition to cancer, low blood count, and birth defects, 6 out of the 10 encounters that discussed risk also covered minor side effects, like rashes and nail changes.

\subsubsection{Uncertainties}

Six of the 14 encounters acknowledged the uncertainties surrounding patient response to hydroxyurea. One physician, for example, conceded that even among patients well suited to take hydroxyurea, reactions differ, noting: 
Example 7

"There are some people who it just sort of changes their life and, and really just makes it so much better, and there are other people who can't tolerate it and it doesn't work." (Encounter 9)

The remaining eight encounters did not mention any uncertainties related to HU.

\subsubsection{Assess Understanding}

The Braddock model describes fostering patient understanding as "the central goal of informed decision making” (p. 2314) and emphasizes the importance of assessing patient understanding. This could mean asking a patient to explain back what was just presented with questions like, “what are some side effects to watch for with hydroxyurea?". In practice, that type of assessment was never fully conducted in any of the 14 encounters. Patient understanding was partially assessed in 3 encounters. In one, understanding was not assessed by the physician, but the patient implied understanding by asking:

\section{Example 8}

"In the event where someone was using [hydroxyurea] consistently and you say it's dropping your white blood cell count-doesn't that open up for more infections?" (Encounter 6). The patient's question demonstrates an understanding of the physician's explanation of hydroxyurea's possible side effects. In the other two encounters, physicians asked their patients if they understood, with questions like "does that make sense?" but did not have patients actively demonstrate understanding (Encounter 8 and Encounter 10).

\subsubsection{Explore Preference}

Exploration of patients' preferences involves physicians asking patients for their opinion to indicate that disagreeing with the physician or asking for more time is also an appropriate course 
of action. Seven encounters did not contain any dialogue related to patient preference and three encounters contained explicit exploration of patient preference. For example, in this encounter, a physician followed up with a patient on a previous conversation and both asked about and acknowledged the patient's preference to decline using hydroxyurea:

Example 9

$\begin{array}{ll}\text { Doctor: } & \begin{array}{l}\text { Last time we were here, we had a long conversation about } \\ \text { hydroxyurea. }\end{array} \\ \text { Patient: } & \text { Yeah, we did. } \\ \text { Doctor: } & \text { And you were going to read up on it. And you decided anything? } \\ \text { Patient: } & \text { Yeah, and I still... } \\ \text { Doctor: } & \text { No? } \\ \text { Patient: } & \text { I'm not comfortable about it. } \\ \text { Doctor: } & \text { Mm-hmm. Mm-hmm. }\end{array}$

(Encounter 11)

The remaining four dialogues fell somewhere in between. One physician explicitly asked for the patient's preference, but the conversation moved away from preference before the patient had a chance to answer (this was not considered fulfilled):

Example 10

Doctor: $\quad$ So what's your take on what you want us to do for you? Because [...] your hemoglobin is low, and usually it's not this low but you see subsequently you have been - it's been getting lower and lower, right? Do you know what your level is normally?

Patient: $\quad U m$, no.

(Encounter 12) 
In three cases, there were conversations where an explicit exploration did not occur because the patient stated her preference unprompted by the provider, or revealed her preference right after the physician stated hers, obviating the need for their physicians to initiate the exploration. The following is an example of a discussion in which the patient states her preference unprompted by the provider:

Example 11

Doctor: [Hydroxyurea] is something that, if you're missing a lot of school - it might be worth - it might be worth that -

Patient: $\quad$ Yeah, I think I want to try that -

Doctor: $\quad$ You want to try that?

(Encounter 10)

\subsection{Patient/Provider Preferences and Discussion Outcomes}

Of the 14 discussions, 5 concluded with concrete plans to start the drug in the future, and 9 left the discussion open.

\section{Figure 1a. Patient decisions among physicians who preferred hydroxyurea}

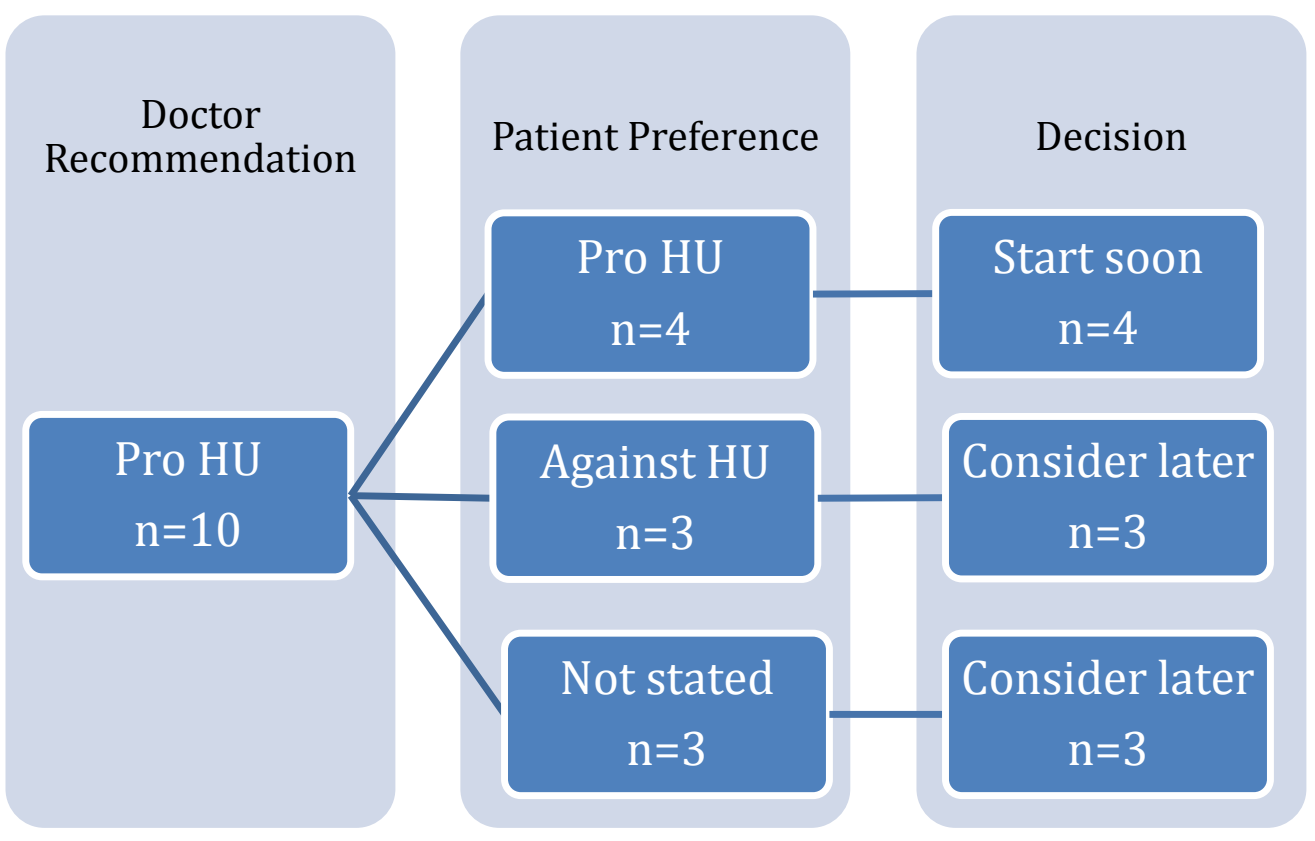


Figure 1b. Patient decisions among physicians ambivalent about hydroxyurea

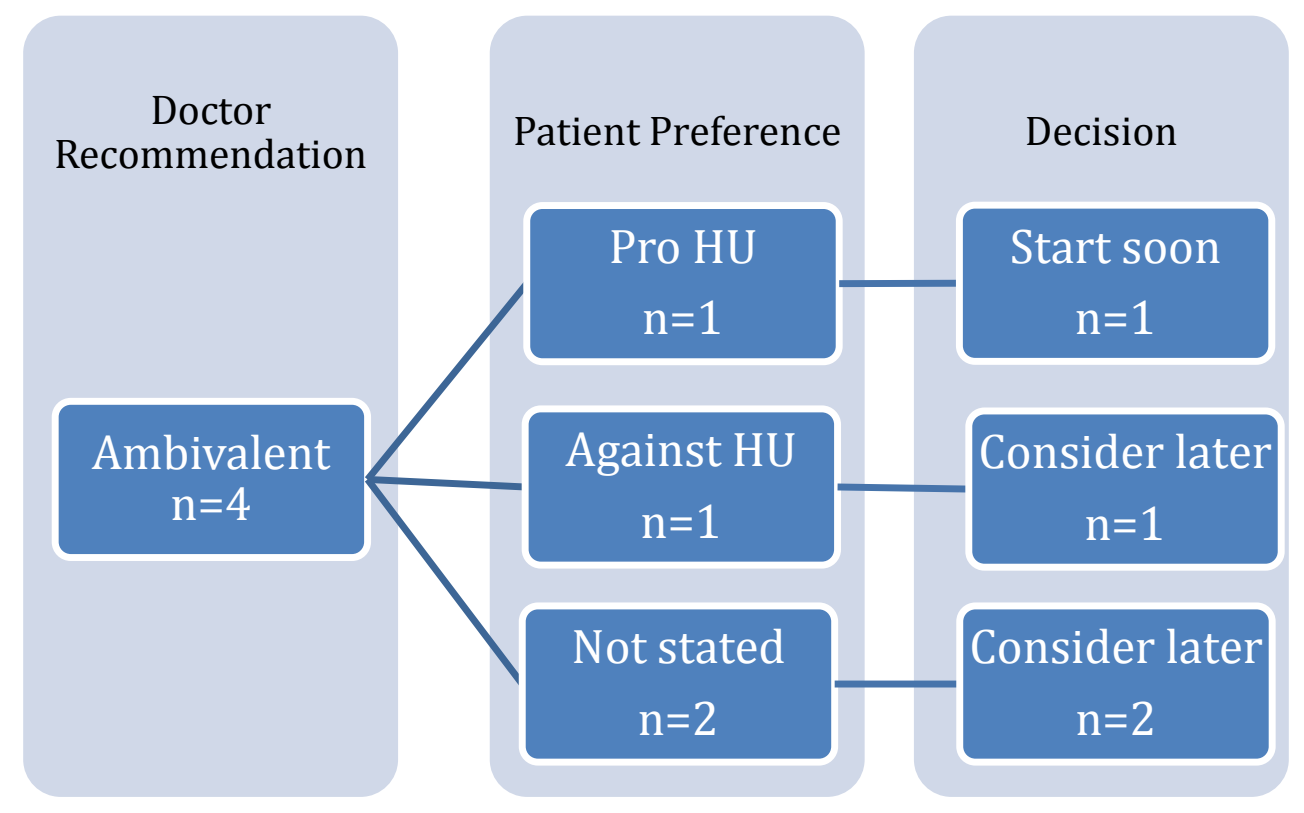

Figure 1 describes these processes and decision outcomes based on the patient and provider preferences ascertained from the dialogue. The decision to initiate hydroxyurea seemed to align more closely with patient preference than with provider preference. None of the patients who preferred not to take hydroxyurea initiated the medication, even if their physicians recommended it, while those patients who indicated that they were pro-HU finished the encounter with either a prescription or planned to get one in the near future.

\subsection{Contribution of Contextual Data}

\subsubsection{Disease Severity}

Figure 2 shows the relationship between disease severity and decision outcome: there was a monotonic increase in the percentage of patients who decided to receive a prescription or made plans to obtain one based on disease severity groups.

Figure 2: Patients who choose to start hydroxyurea, by number of crises 


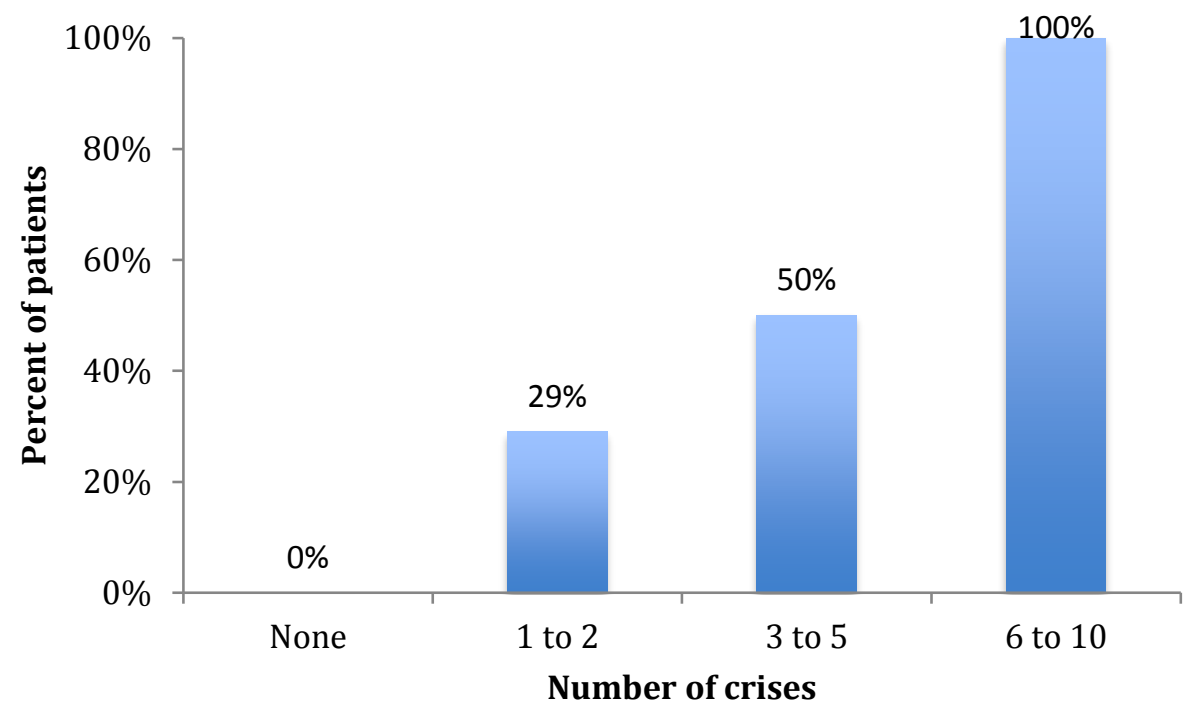

The severity of the five patients who received a prescription or had concrete plans to obtain a prescription in the future ranged from those having painful crises 1-2 times in the past year (2 of 9 patients with crisis 1-2 times per year chose to start HU), to 3-5 crises ( 2 of 4 patients chose $\mathrm{HU}$ ), to 6-10 crises (the only patient in our sample with 6-10 crises per year chose to start HU).

While patient decisions regarding hydroxyurea differed by disease severity, the way physicians discussed hydroxyurea did not appear to differ by disease severity—elements of Braddock's model of shared decision making were equally present in the non-severe group (1-2 crises a year) as in the severe group ( 3 or more crises a year). Both groups averaged 3.42 Braddock elements per encounter.

\subsubsection{Patient-reported Concerns about Hydroxyurea}

Most of the patients $(\mathrm{n}=11)$ indicated that a doctor had previously recommended the drug to them on the baseline survey, after which the survey asked about the concerns they might have with hydroxyurea. Nine patients expressed some sort of concern about hydroxyurea.

Of these concerns, worries about side effects were the most commonly reported (7/11). The concern reported on the questionnaire was fully addressed in 6 of the 7 patient-physician 
encounters. The patients raised side effect concerns in 3 encounters and the physicians responded by discussing the risks of hydroxyurea initiation. In 3 of the encounters, the physicians talked about the risks though the patients did not raise their concerns to the physicians. In the one encounter where the patient's concern was only partially addressed, the physician did not explicitly discuss side effects and the patient did not raise the issue, but the physician did discuss what it would be like to take the medication and the monitoring required while taking the drug. The same patient also indicated not wanting to take a medicine every day. Again, the physician did not explicitly address this concern, nor did the patient bring it up in the encounter.

One patient expressed concern that hydroxyurea was an experimental drug. While the encounter does not capture the patient raising this concern, the physician did address it, presenting a thorough history of the use of hydroxyurea and indicating that it has been FDAapproved for SCD since 1998.

One patient indicated in the questionnaire a concern that hydroxyurea would not help. Although this patient did not explicitly raise the issue in the conversation, it was shared by the physician, who said to the patient, "if I felt strongly [about you taking] hydroxyurea, you would know it." Finally, two patients indicated they were concerned about hydroxyurea use because they were thinking about having children—for one patient, their physician fully addressed the issue and discussed how initiation might affect having a child. For the other patient, the physician did not address the concern, nor did the patient mention it explicitly.

\section{Discussion and conclusion}

\subsection{Discussion}


In this analysis of dialogues between physicians and patients with a serious chronic illness, we observed a spectrum of shared decision-making behaviors by physicians. While there were certain elements that physicians routinely discussed, like the clinical scenario and the benefits of taking the medication, there were other elements such as the patient's role in making the decision and assessment of patient understanding that never occurred. Nevertheless, although physicians did not fully discuss all the elements of the SDM framework, patients did seem to know their role and displayed their active agency in the decision process as the final decisions about hydroxyurea were more aligned with their own stated preferences than those of their physicians. These findings raise questions of what 'counts' as shared decision making, provide insight into what occurs in practice, and also point to areas for physician improvement.

While risks of treatment were discussed in most encounters, there was not one uniform approach to which particular risks got mentioned or how physicians presented them. Such variation seemed to result from the informal nature of conversations, and physicians who rely on their memory to outline some part of the full spectrum of risks while talking to patients. This suggests that standardized decision aids may be helpful, which can detail the risks and benefits of hydroxyurea to fully inform all patients and help them understand the nuances of initiation. A standardized decision aid, one that lays out all possible risks and benefits, could help physicians provide all the relevant and necessary information to patients, and help patient start a discussion about their own particular concerns (Trikalinos et al. 2014; Volk et al. 2016; Stacey et al. 2017). Such aids have been shown to be effective in improving patient-doctor communication in a number of settings, from cancer screening to the initiation of arthritis medication (Herrmann et al. 2016; Nathan et al. 2016; Nota et al. 2016). 
Interventions such as decision aids or pre-visit agenda forms would also help address another deficiency uncovered by our study, which had contextual information about patient concerns - which was that they often were not discussed or brought up by the patient. Although we did not collect post-encounter data from the patient to assess how the patient felt about their concerns not being addressed, we hypothesize that these un-discussed concerns about hydroxyurea might create some resistance or confusion about whether to take the medication. Beyond decision aids, other standardized processes, such as having patients complete agenda forms to present to their physicians, or having physicians ask about their patients' agenda at the beginning of a visit, may also help physicians address patient concerns. In one analysis, Dyche et al. noted that physicians who did not ask about their patients' concerns were less likely to correctly identify their patients' needs (Dyche and Swiderski 2005). While addressing the patients' agendas may lead to longer visits, building such practices into the encounter was also associated with greater patient satisfaction (Hornberger et al. 1997; Wilson et al. 2012; Middleton et al. 2006)

More than a sense of respect or involvement by patients, shared decision making has been associated with positive health outcomes, like medication adherence. In a study comparing clinician decision making and shared decision making with usual care among patients with asthma, Wilson et al. (2012) found that shared decision making significantly improves patient adherence to pharmacotherapy as well as clinical outcomes. Similarly, Bauer et al. (2014) found that the lack of shared decision making was associated with antidepressant non-adherence . Although this analysis did not examine patient adherence and other outcomes post-decision making, the effectiveness of shared decision making is highly relevant to this patient and disease population. Anecdotal stories in this population suggest that many patients have preconceived 
ideas about hydroxyurea and that no matter what the clinician says, they are not going to take the medication, but the effectiveness of SDM in other patient populations suggests it could be effective here as well.

This analysis identified several areas of shared decision making future improvement efforts could focus on, including the discussion of pros and cons, the patient's role, and an assessment of patient understanding. While decision aids and standardized processes aim to facilitate patients' informed engagement with healthcare in the context of their lives, shared decision making interventions aim to facilitate the conversation between patients and professionals. Previous research on SDM training for health professionals has demonstrated the effectiveness of SDM training on improving general SDM competencies as well as general patient interaction competencies (Bieber et al. 2009). Future work may do well in focusing on those specific elements of SDM while overlooking others.

While SDM is an important aspect of patient-provider communication, some have suggested that it might not be needed in every clinical encounter. Whitney et al. describe SDM as being morally relevant only under conditions of clinical uncertainty (Whitney et al. 2004). The use of hydroxyurea could be considered clearly indicated (high certainty) for patients with severe SCD and somewhat more controversial (lower certainty) for those with low disease severity. If physicians were following this framework, one would have expected to see the way physicians approach shared decision making to be different between the two groups. Yet the encounters we observed did not bear out this way. Although more severe disease patients chose to start hydroxyurea, the elements of SDM were equally present in the severe disease group as in the less severe group. 


\subsection{Conclusion}

This analysis offers implications and opportunities for improvement for several key stakeholders, including patients, physicians, and researchers. While this study was limited by a small sample size of only fourteen encounters, this exploratory and hypothesis-generating study on shared decision making has led to valuable observations that can inform medical practice. Patients can be empowered to know their agency in the decision process and also find ways to remember and bring up their concerns during medical visits. Physicians should ask more questions about patients' concerns, preferences, and understanding of the situation.

For communication researchers and the academic community more broadly, we might reconsider how elements of SDM could be measured to capture a realistic view of what 'counts' as SDM. Explicit statements about the patient's role in decision making may not be as morally necessary in contexts where patients are refusing treatment as, in these cases, patients seem to know their role. Health systems might consider the creation of more standardized decision aids to help physicians deal with the complexities of treatments that need to be explained in shorter visit times, and ensure that patients get the information they need to make good decisions.

Acknowledgement: The study was funded by NIH grant R01HL088511. In addition, Dr. Beach was funded by K24 DA037804.

Conflict of Interest: The authors have no conflicts of interest to declare. 
References

Bauer, A. M., Parker, M. M., Schillinger, D. et al. (2014) Associations between antidepressant adherence and shared decision-making, patient-provider trust, and communication among adults with diabetes: Diabetes study of Northern California (DISTANCE). Journal of General Internal Medicine 29 (8): 1139-1147. doi:10.1007/s11606-014-2845-6.

Bieber, C., Nicolai, J., Hartmann, M. et al. (2009) Training physicians in shared decisionmaking? Who can be reached and what is achieved? Patient Education and Counseling 77 (1): $48-54$.

Braddock, C. H., Edwards, K. A., Hasenberg, N. M., Laidley, T. L. and Levinson, W. (1999) Informed decision making in outpatient practice: Time to get back to basics. The Journal of the American Medical Association 282 (24): 2313-2320.

Collins, S., Drew, P., Watt, I. and Entwistle, V. (2005) 'Unilateral' and 'bilateral' practitioner approaches in decision-making about treatment. Social Science \& Medicine 61 (12): 26112627. doi:10.1016/j.socscimed.2005.04.047.

Dyche, L. and Swiderski, D. (2005) The effect of physician solicitation approaches on ability to identify patient concerns. Journal of General Internal Medicine 20 (3): 267-270. doi:10.1111/j.1525-1497.2005.40266.x.

Elwyn, G., Edwards, A., Mowle, S. et al. (2001) Measuring the involvement of patients in shared decision-making: A systematic review of instruments. Patient Education and Counseling 43 (1): 5-22.

Elwyn, G., Frosch, D., Thomson, R. et al. (2012) Shared decision making: A model for clinical practice. Journal of General Internal Medicine 27 (10): 1361-1367. doi:10.1007/s11606012-2077-6.

Gulbrandsen, P., Dalby, A. M. L., Ofstad, E. H. and Gerwing, J. (2014) Confusion in and about shared decision making in hospital outpatient encounters. Patient Education and Counseling 96 (3): 287-294. doi:10.1016/j.pec.2014.07.012.

Herrmann, A., Mansfield, E., Hall, A. E., Sanson-Fisher, R. and Zdenkowski, N. (2016) Wilfully out of sight? A literature review on the effectiveness of cancer-related decision aids and implementation strategies. BMC Medical Informatics and Decision Making 16 (1): 36. doi:10.1186/s12911-016-0273-8.

Hornberger, J., Thom, D. and MaCurdy, T. (1997) Effects of a self-administered previsit questionnaire to enhance awareness of patient's concerns in primary care. Journal of General Internal Medicine 12 (10): 597-606. doi:10.1046/j.1525-1497.1997.07119.x. 
Landmark, A. M. D., Svennevig, J. and Gulbrandsen, P. (2016) Negotiating treatment preferences: Physicians' formulations of patients" stance. Social Science \& Medicine 149: 26-36. doi:10.1016/j.socscimed.2015.11.035.

Matthias, M. S., Salyers, M. P. and Frankel, R. M. (2013) Re-thinking shared decision-making: Context matters. Patient Education and Counseling 91 (2): 176-179. doi:10.1016/j.pec.2013.01.006.

Mendick, N., Young, B., Holcombe, C, and Salmon, P. (2010) The ethics of responsibility and ownership in decision-making about treatment for breast cancer: Triangulation of consultation with patient and surgeon perspectives. Social Science \& Medicine 70 (12): 1904-1911. doi:10.1016/j.socscimed.2009.12.039.

Middleton, J. F., McKinley, R. K. and Gillies, C. L. (2006) Effect of patient completed agenda forms and doctors' education about the agenda on the outcome of consultations: Randomised controlled trial. British Medical Journal 332 (7552): 1238-1242. doi:10.1136/bmj.38841.444861.7C.

Nathan, A. G., Marshall, I. M., Cooper, J. M. and Huang, E. S. (2016) Use of decision aids with minority patients: A systematic review. Journal of General Internal Medicine 31 (6): 663676. doi:10.1007/s11606-016-3609-2.

National Heart, Lung, and Blood Institute (2014) Evidence-Based Management of Sickle Cell Disease. Retrieved from https://www.nhlbi.nih.gov/sites/www.nhlbi.nih.gov/files/sicklecell-disease-report\%20020816.pdf.

Nota, I., Drossaert, C. H. C., Taal, E., Vonkeman, H. E., Haagsma, C. J. and van de Laar, M. A. F. J. (2016) Evaluation of a patient decision aid for initiating disease modifying antirheumatic drugs. Arthritis Research \& Therapy 18 (1): 252. doi:10.1186/s13075-016-11383.

Pilnick, A. and Dingwall, R. (2011) On the remarkable persistence of asymmetry in doctor/patient interaction: A critical review. Social Science \& Medicine 72 (8): 1374-1382.

Pilnick, A. and Zayts, O. (2016) Advice, authority and autonomy in shared decision-making in antenatal screening: The importance of context. Sociology of Health \& Illness 38 (3): 343359. doi:10.1111/1467-9566.12346.

Robertson, M., Moir, J., Skelton, J., Dowell, J. and Cowan, S. (2011) When the business of sharing treatment decisions is not the same as shared decision making: A discourse analysis of decision sharing in general practice. Health 15 (1): 78-95. doi:10.1177/1363459309360788.

Stacey, D., Légaré, F., Lewis, K. et al. (2017) Decision aids for people facing health treatment or screening decisions. In D. Stacey (ed) Cochrane Database of Systematic Reviews, 4 (9): CD001431. doi:10.1002/14651858.CD001431.pub5. 
Trikalinos, T. A., Wieland, L. S., Adam, G. P., Zgodic, A. and Ntzani, E. E. (2014) Decision aids for cancer screening and treatment. AHRQ Comparative Effectiveness Reviews 15EHC002-EF

Volk, R. J., Linder, S. K., Lopez-Olivo, M. A. et al. (2016) Patient decision aids for colorectal cancer screening. American Journal of Preventive Medicine 51 (5): 779-791.

Whitney, S. N., McGuire, A. L. and McCullough, L. B. (2004) A typology of shared decision making, informed consent, and simple consent. Annals of Internal Medicine 140 (1): 5459. doi:10.7326/0003-4819-140-1-200401060-00012.

Wilson, S. R., Strub, P., Buist, A.S. et al. (2012) Shared treatment decision making improves adherence and outcomes in poorly controlled asthma. American Journal of Respiratory and Critical Care Medicine 181 (6): 566-577. doi:10.1164/rccm.200906-0907OC. 